\title{
Ising exponents in the two-dimensional site-diluted Ising model
}

\author{
H. G. Ballesteros*, L. A. Fernández ${ }^{\dagger}$, \\ V. Martín-Mayor ${ }^{\ddagger}$, A. Muñoz Sudupe ${ }^{\S}$, \\ Departamento de Física Teórica I, Facultad de CC. Físicas, \\ Universidad Complutense de Madrid, 28040 Madrid, Spain. \\ G. Parisi ${ }^{\uparrow}$ and J. J. Ruiz-Lorenzo".
Dipartimento di Fisica and Istituto Nazionale di Fisica Nucleare,
Università di Roma "La Sapienza", P. A. Moro 2, 00185 Roma, Italy. \\ July 17, 1997
}

\begin{abstract}
We study the site-diluted Ising model in two dimensions with Monte Carlo simulations. Using finite-size scaling techniques we compute the critical exponents observing deviations from the pure Ising ones. The differences can be explained as the effects of logarithmic corrections, without requiring to change the Universality Class.
\end{abstract}

Key words: Lattice. Monte Carlo. Disordered Systems. Critical exponents. Finite-size scaling. $\epsilon$-expansion.

PACS: 05.50.+q;05.70.Jk;11.10.Kk;75.10.Nr;75.40.Mg

\footnotetext{
*hector@lattice.fis.ucm.es

$\dagger$ laf@lattice.fis.ucm.es

$\ddagger$ victor@lattice.fis.ucm.es

$\S$ sudupe@lattice.fis.ucm.es

Tgiorgio.parisi@roma1.infn.it

$\|_{\text {ruiz@chimera.roma1.infn.it }}$
} 
Recently [1], it has been reported using Monte Carlo simulations, that the site-diluted Ising model in two dimensions seems to present a second order transition line with concentration dependent critical indices. Previously, other authors [2, 3] had claimed, using analytical methods, an Ising critical behavior corrected with logarithms.

The field theoretical predictions for this model (based on Renormalization Group and Conformal Field Theory) [4] assesses that disorder does not change the $\eta$ or $\nu$ exponents since it only changes subleading terms. However, the specific heat, at the critical point, diverges as $\log (\log L)$ in the disordered system, while it does as $\log L$ in the pure case $L$ being the lattice size.

In this letter, we extend the methods developed in ref. [5] for the four dimensional site-diluted Ising model to the two-dimensional case.

We observe that, although an apparent variation of the indices seems to happen when varying the concentration, this can be explained as a transient effect. In fact, a pure Ising value for the indices plus logarithmic corrections fits very well our Monte Carlo (MC) data. When preparing this letter, other authors have also reported similar conclusions in the bond diluted Ising model using different techniques [6].

The numerical methods as well as the analytical computations based on the perturbative renormalization group are very similar in four and two dimensions, so we will report here just the minimal details required, centering ourselves in the description of the results. We address to reference [5] for further details of the method.

Our procedure is based on a Finite-Size Scaling (FSS) analysis. We perform MC simulations in the critical region for several values of the concentration $p$. For each $p$ value, we generate hole configurations in a square lattice filling the sites randomly with probability $p$.

For each hole configuration (sample), we perform a MC simulation of the Ising model defined as the set of spins lying in the filled sites coupled through a nearest-neighbor interaction. In the smallest concentration we use the cluster Swendsen-Wang algorithm [7] to update the signs of the spins. For the rest of concentrations, we found the Wolff single cluster version of this algorithm [8] to be more efficient. At each sample, we measure the energy, the magnetization and the Fourier transform of the Ising field at minimal momenta in about 100 independent spin configurations. We store the independent measures in order to compute derivatives with respect to the coupling and to extrapolate the results to close values of the coupling and the dilution. We have extrapolated in $\beta$ in all cases but $p \simeq 2 / 3$ where 


\begin{tabular}{|r|l|l|l|l|}
\hline$L$ & $p=1.0$ & $p=0.88889$ & $p=0.75$ & $p=0.6666$ \\
\hline \hline 24 & $1.009(4)$ & & & \\
\hline 32 & $1.009(5)$ & $1.078(6)$ & $1.142(9)$ & $1.182(12)$ \\
\hline 48 & $0.995(4)$ & $1.072(6)$ & $1.114(9)$ & $1.170(12)$ \\
\hline 64 & $1.001(4)$ & $1.079(5)$ & $1.112(9)$ & $1.151(10)$ \\
\hline 96 & $1.010(5)$ & $1.075(6)$ & $1.111(9)$ & $1.125(12)$ \\
\hline 128 & $0.993(4)$ & $1.066(5)$ & $1.098(8)$ & $1.141(13)$ \\
\hline 192 & & $1.065(6)$ & $1.096(9)$ & $1.140(13)$ \\
\hline 256 & $1.004(5)$ & & & \\
\hline
\end{tabular}

Table 1: The $\nu$ exponent for $(L, 2 L)$ pairs at different dilutions.

a $p$ extrapolation performs better.

To reduce statistical errors, we have generated 10,000 hole samples for each $(L, p)$ pair, $L$ being the lattice size. We have simulated at concentrations: $p=1,8 / 9,3 / 5,2 / 3$. Let us remember that the percolation threshold is at $p_{\mathrm{c}} \simeq 0.59$ [9]. The FSS method that we use [10] is based upon the ratio of several observables: magnetization, susceptibility, correlation length, Binder cumulants, and their derivatives for two different lattice sizes $L_{1}$ and $L_{2}$. At the parameter values where the correlation lengths ratio matches $L_{2} / L_{1}$ we expect that, in the absence of logarithmic corrections

$$
\frac{O\left(L_{2}, \beta, p\right)}{O\left(L_{1}, \beta, p\right)}=\left(L_{2} / L_{1}\right)^{x_{O} / \nu}+O\left(L_{1}^{-\omega}\right),
$$

where $x_{O}$ is the critical exponent for the observable $O$, e.g. $\gamma$ for the susceptibility, $\nu$ for the correlation length, etc. $\omega$ is the universal correctionsto-scaling exponent. The $\beta$-derivatives of the previous observables go with the corresponding exponents plus $1 / \nu$. In all cases we use pairs of lattices of sizes $L$ and $2 L$.

In table 1 and 2 we report the results for the exponents $\nu$ and $\eta$ respectively, using the relation (11) for the $\beta$-derivative of the correlation length in the former case and the susceptibility in the latter (after applying the scaling relation $\eta=2-\gamma / \nu)$. Notice that there is some statistical anticorrelation between sucessive even (odd) rows. We recall the critical exponents in the $\beta \rightarrow \infty$ limit (pure site percolation), $\nu=4 / 3$ and $\eta=5 / 24$ conjectured by Nienhuis 11] and in agreement with a recent MC work 12.

Although the $\nu$ exponent seems to be non-constant as a function of the concentration, the values for $\eta$ are very stable. This fact was also observed 


\begin{tabular}{|r|c|l|l|l|}
\hline$L$ & $p=1.0$ & $p=0.88889$ & $p=0.75$ & $p=0.6666$ \\
\hline \hline 24 & $0.2465(7)$ & & & \\
\hline 32 & $0.2466(8)$ & $0.2495(9)$ & $0.2504(10)$ & $0.2454(18)$ \\
\hline 48 & $0.2495(8)$ & $0.2487(7)$ & $0.2498(11)$ & $0.2448(14)$ \\
\hline 64 & $0.2499(8)$ & $0.2490(8)$ & $0.2469(8)$ & $0.2455(15)$ \\
\hline 96 & $0.2498(8)$ & $0.2501(8)$ & $0.2460(9)$ & $0.2456(14)$ \\
\hline 128 & $0.2497(8)$ & $0.2495(8)$ & $0.2469(9)$ & $0.2461(13)$ \\
\hline 192 & & $0.2485(8)$ & $0.2483(7)$ & $0.2483(14)$ \\
\hline 256 & $0.2517(7)$ & & & \\
\hline
\end{tabular}

Table 2: The $\eta$ exponent for $(L, 2 L)$ pairs at different dilutions.

\begin{tabular}{|l|l|c|c|c|}
\hline \multicolumn{1}{|c|}{$p_{\mathrm{c}}$} & \multicolumn{1}{c|}{$\beta_{\mathrm{c}}$} & $\omega$ & $\chi^{2} /$ d.o.f. & $L_{1}-\left(L_{2}^{a}-L_{2}^{b}\right)$ \\
\hline \hline 1 & $0.440682(5)$ & $1.5(8)$ & $15.2 / 12$ & $24-(32-512)$ \\
\hline 0.88889 & $0.53781(2)$ & $1.0(3)$ & $2.6 / 8$ & $48-(64-384)$ \\
\hline 0.75 & $0.77125(8)$ & $0.9(5)$ & $7.7 / 6$ & $48-(96-384)$ \\
\hline $0.66661(3)$ & 1.10 & $0.6(2)$ & $9.6 / 8$ & $32-(64-384)$ \\
\hline
\end{tabular}

Table 3: Critical parameters for several dilutions. We also show the computed corrections to scaling exponent $\omega$, the fit quality and the range of lattice sizes used (crossings from $L_{1}-L_{2}^{a}$ to $L_{1}-L_{2}^{b}$ ).

in the scaling of the Yang-Lee zeroes at the critical point 13.

An interpretation of these results could be a continuous set of Universality Classes, as assumed in reference [1]. However, a simpler scenario is a fixed value for $\nu$ (that of the Ising Model) plus logarithmic corrections.

Using the results from refs. [2, 14] it is possible to show that the derivative of the correlation length with $\beta$ reads at critical point as

$$
\partial_{\beta} \xi \propto-\frac{\xi^{2}}{\sqrt{1+C \log L}}\left(1+\frac{C}{2+2 C \log L}\right)
$$

where $C$ depends on the dilution. Computing the $\nu$ exponent from (11) and (2) we obtain an apparent behavior as

$$
\nu^{\text {apparent }}=1+A / \log L+\ldots
$$

In figure 1 we plot the values of $\nu$ reported in table 2 as a function of $1 / \log L$. All our MC data are compatible with a behavior of type (3). 


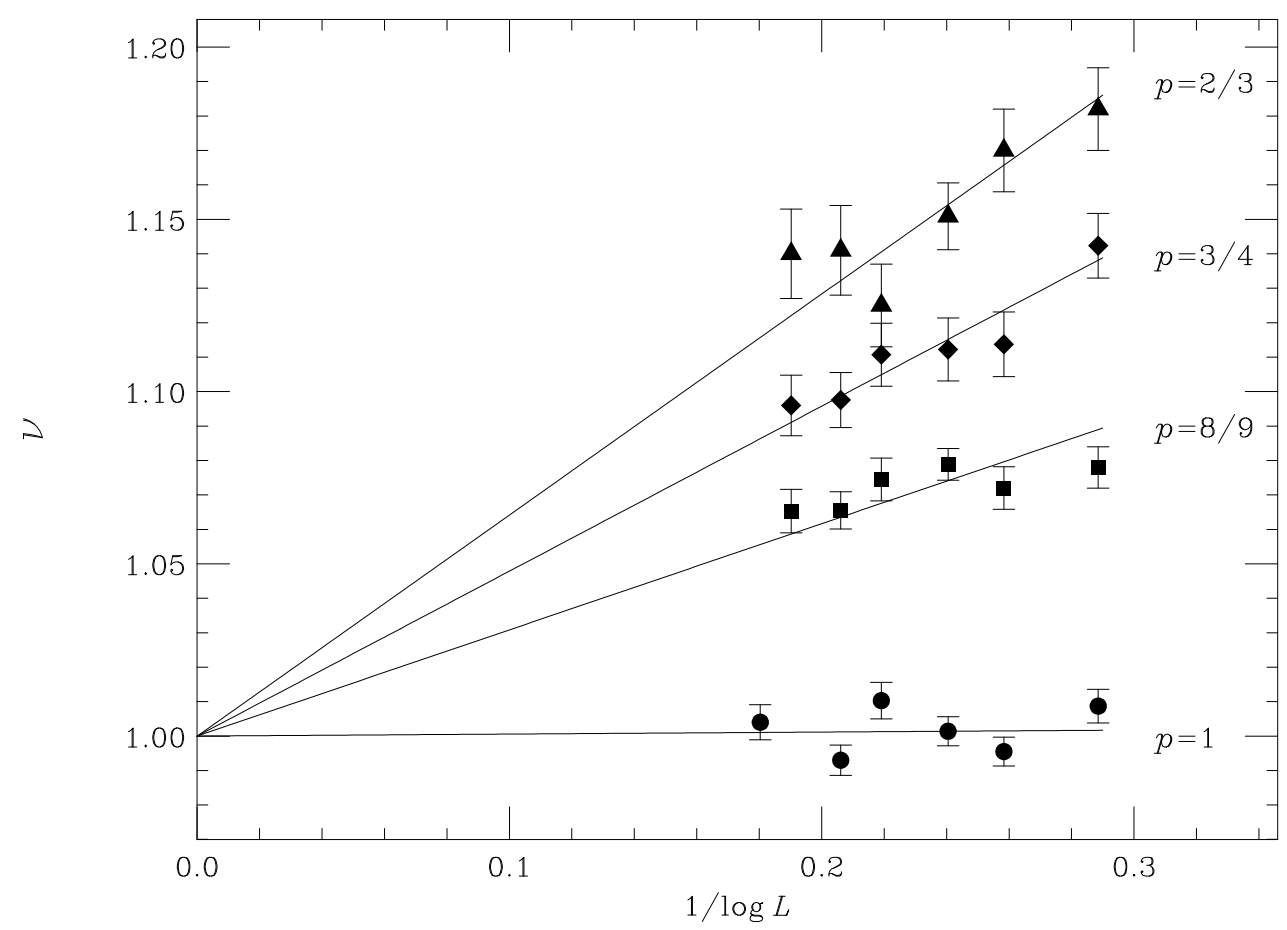

Figure 1: $\nu$ exponent as a function of $1 / \log L$ for several values of $p$.

The critical parameters can be computed with great accuracy studying the crossing of several quantities (second momentum correlation length divided by the lattice length, and Binder parameter for the magnetization) for the different lattice sizes. The deviation of the crossing point for lattices sizes $L, s L$ scales as

$$
\Delta \beta_{\mathrm{c}}^{L}, \Delta p_{\mathrm{c}}^{L} \propto \frac{1-s^{-\omega}}{s^{1 / \nu}-1} L^{-\omega-1 / \nu},
$$

We address to reference 10 for details of the method. The results are reported in table 3. Notice that for the Ising Model the method gives the correct value within errors for the critical temperature (1 part in $\left.10^{5}\right)$. Regarding the $\omega$ exponent, whose conjectured value is $\omega=4 / 3$ [15] for the pure system, to obtain more accurate results, simulations on smaller lattices should be added, but it would increase the systematic errors in the determination of the critical coupling.

We finally measure the specific heat $C_{V}$ at the critical points. The results 

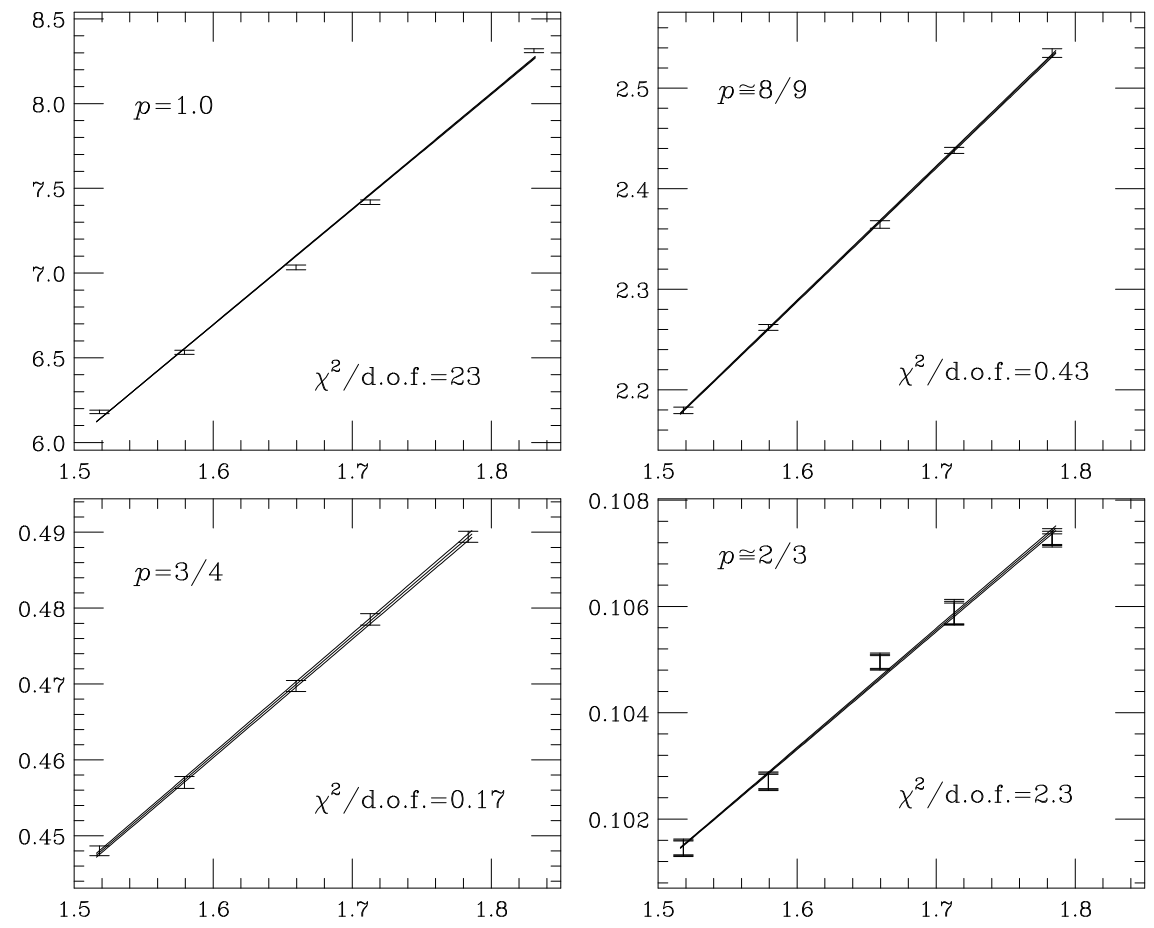

Figure 2: Specific heat as a function of the double logarithm of the lattice size. The lines correspond to linear fits for $L \geq 96$. The three lines for each case show the effect of the error in the determination of the critical parameters.

are displayed in figure 2. Excluding the Ising limit, a linear function of $\log (\log L)$, as predicted in ref. [2], fits very well the data.

We thus conclude that the site diluted Ising model in two dimensions belongs to the same Universality Class as the pure Ising model, although we find strong logarithmic effects.

We thank to the CICyT (contracts AEN94-0218, AEN96-1634) for partial financial support. JJRL is granted by EC HMC (ERBFMBICT950429).

\section{References}

[1] J.K. Kim and A. Patrascioiu, Phys. Rev. Lett. 72 (1994) 2785; Phys. Rev. B49 (1994) 15764. 
[2] V.S. Dotsenko and Vi. S. Dotsenko, Adv. Phys. 32 (1983) 129.

[3] V.B. Andreichenko, Vi.S. Dotsenko, W. Selke and J. S. Wang. Nucl. Phys. B344 (1990) 531.

[4] V. Dotsenko, M. Picco and P. Pujol, "Correlation functions for the 2D random bonds Potts Models". Proceedings of the Trieste Conference on Recent Developments in Statistical Mechanics and Quantum Field Theory. cond-mat/9509149.

[5] H.G. Ballesteros, L.A. Fernández, V. Martín-Mayor, A. Muñoz Sudupe, G. Parisi, J.J. Ruiz-Lorenzo, hep-lat/9707017.

[6] F. D. A. Aarão Reis, S. L. A. de Queiroz and R. R. dos Santos, cond-mat 9706202 .

[7] R. H. Swendsen and J. S. Wang, Phys. Rev. Lett. 58 (1987) 86.

[8] U. Wolff, Phys. Rev. Lett. 62 (1989) 3834.

[9] D. Stauffer and A. Aharony. Introduction to the percolation theory. (Taylor \& Francis, London 1994)

[10] H. G. Ballesteros, L.A. Fernández, V. Martín-Mayor, and A. Muñoz Sudupe, Phys. Lett. B378 (1996) 207; Phys. Lett. B387 (1996) 125; Nucl. Phys. B 483 (1997) 707.

[11] B. Nienhuis, J. Phys. A: Math. Gen. 15 (1982) 199.

[12] H.G. Ballesteros, L.A. Fernández, V. Martín-Mayor, A. Muñoz Sudupe, G. Parisi, J.J. Ruiz-Lorenzo, Phys. Lett. B400 (1997) 346.

[13] J. J. Ruiz-Lorenzo, J. Phys. A30 (1997) 485.

[14] B. N. Shalaev, Sov. Phys. Solid State 26 (1984) 1811; R. Shankar, Phys. Rev. Lett. 58 (1987) 2466.

[15] J. Zinn-Justin, Quantum Field Theory and Critical Phenomena (Oxford Science Publications 1990). 